\title{
Características de los Sistemas Centrales de Conocimiento en niños de 3 a 6 años de edad $^{*}$
}

\section{Ontogenetic characteristics of sex, age and socioeconomic status in the Core Knowledge Systems in 3 to 6 years-old Children}

Recepción: 21 Mayo 2018 | Aceptación: 05 Junio 2018

\author{
María Fernanda Rueda-Posada \\ Universidad de la Sabana, Colombia \\ ORCID: http://orcid.org/0000-0002-7939-828X \\ María Fernanda Quiroz-Padilla \\ Universidad de la Sabana, Colombia \\ ORCID: http://orcid.org/0000-0001-9425-2564 \\ JuAn José Giraldo-HuErtas ${ }^{a}$ \\ Universidad de la Sabana, Colombia \\ ORCID: http://orcid.org/0000-0002-9841-0336
}

a Autor de correspondencia: Correo electrónico:
juangh@unisabana.edu.co

Para citar este artículo: Rueda-Posada, M. F., QuirozPadilla, M. F., \& Giraldo-Huertas, J. J. (2018). Características ontogenéticas de sexo, edad y estrato socioeconómico en los Sistemas Centrales de Conocimiento en niños de 3 a 6 años de edad. Universitas Psychologica, 17(5), 1-14. http:// doi.org/10.11144/Javeriana.upsy17-5.cscc

\begin{abstract}
RESUMEN
Los Sistemas Centrales de Conocimiento son la base de las habilidades cognitivas de la especie humana. Teniendo en cuenta el valor evolutivo de los mismos, se buscó reconocer las relaciones o diferencias entre estos y otras variables de crecimiento (sexo y edad) y variables ambientales (nivel socioeconómico). Para ello, se evaluó cada sistema central de conocimiento y el desarrollo sociocognitivo de 164 niños y 164 niñas, entre los 37 y 71 meses de edad $(M=54$ meses; $\mathrm{DE}=0.55)$. $\mathrm{Al}$ aplicar una prueba Kruskal-Wallis se encontró que la edad tuvo un efecto significativo sobre el índice general de desarrollo sociocognitivo $(\mathrm{p}<$ 0.001) y sobre el reconocimiento funcional del objeto $\left(\chi^{2}=54.221, \mathrm{p}\right.$ $<0.001)$, del número $(\chi 2=85.735, \mathrm{p}<0.001)$ y la ubicación espacial $\left(\chi^{2}=8.258, \mathrm{p}<0.016\right)$. En contraste, no se hallaron efectos del sexo ni del nivel socioeconómico para las diferencias en los sistemas centrales de conocimiento ni en el índice de desarrollo sociocognitivo.

Palabras clave

sistemas centrales de conocimiento; desarrollo cognitivo; cognición numérica; cognición social; psicología del aprendizaje.
\end{abstract}

\section{ABSTRACT}

Core knowledge systems are the basis of our capacity to construct and learn new abilities and concepts. The objective of this study was to identify possible relationships and differences between Core knowledge systems, depending on variables of age, sex and parental socioeconomic status. This was done using an evaluation of socio-cognitive development in 328 participants (164 girls, 164 boys) with an age range between 37 and 71 months $(\mathrm{M}=54$ months, $\mathrm{SD}=0.55)$ and the application of one task for each core knowledge system. A Kruskal-Wallis test revealed an effect of age on achievement in the general index of socio-cognitive development $(\mathrm{p}<0.001)$, and this difference was also found in the tasks of functional object recognition $(\chi 2=54.2, p<0.001)$, numerical cognition 
$\left(\chi^{2}=85.7, \mathrm{p}<0.001\right)$, and spatial location $\left(\chi^{2}=8.26, \mathrm{p}\right.$ $=0.016)$. No differences were found in relation to sex or socioeconomic status.

Keywords

core knowledge systems; executive functions; cognitive development; psychology of learning.

Históricamente, se pueden rastrear al menos tres escuelas diferentes (MacDonald \& Hershberger, 2005) que han orientado la manera como filosofía y ciencia pueden llegar a comprender los procesos relacionados con el conocimiento y el desarrollo humano. Estas tres perspectivas, innatismo, empirismo y evolucionismo, han provocado durante décadas la generación de modelos y descubrimientos sobre cómo adquirimos y utilizamos el conocimiento (Palacios, 1990).

En el marco de la perspectiva evolucionista, se desprende una corriente de interés por el análisis de diferentes eventos de percepción, captura y procesamiento de la información, y se gesta la tesis modularista de Fodor (1983), según la cual el funcionamiento cognitivo es un conjunto de módulos encapsulados cada uno especializado y bajo un dominio concreto de información, a partir del cual, se forman representaciones con las que opera un sistema central de ejecución cognitiva (Fodor, 1983; MacDonald \& Hershberger, 2005). Esta propuesta, aunque criticada y contrarrestada por análisis del grado de integración que pueden alcanzar diversos sistemas para diversos conocimientos (Rips, 2017), ha tenido gran influencia en la perspectiva del desarrollo de habilidades y conocimientos, como por ejemplo la teoría de redescripción representacional de Karmiloft-Smith et al. (Karmiloff-Smith, Crespo, \& Bernardos, 1994). Pero aún más, sobre los trabajos de Spelke y cols. (Spelke, 1990; Spelke, 2000, 2005; Spelke \& Kinzler, 2007; Spelke, Lee, \& Izard, 2010) quienes, con base en una serie de experimentos con infantes humanos y otras especies, plantean la teoría de los "Core Knowledge Systems", en adelante, Sistemas centrales de Conocimiento (SCC) (Spelke, 2000; Spelke \& Kinzler, 2007). Estos sistemas que serán descritos a continuación, requieren para la literatura reciente sobre ellos (Barner \& Baron, 2016; Rips, 2017) dos respuestas que se intentan responder en este artículo de manera limitada: 1$)$ se integran adecuadamente durante el desarrollo cognitivo individual y 2) se ven afectados por variables tanto ambientales cómo genéticas de forma continua durante el desarrollo individual.

\section{Los sistemas centrales de conocimiento (SCC)}

Los seres humanos están dotados innatamente con los cimentos básicos sobre los cuales se construye el conocimiento y la forma de comprender la información, es decir, las cantidades, las formas, las interacciones sociales, etc. (Gauvrit, Zenil, Soler-Toscano, Delahaye, \& Brugger, 2017). Spelke (2000) propone los sistemas centrales o nucleares de conocimiento como la base de futuras construcciones en la forma de comprender o conocer el entorno. Los SCC funcionan como mecanismos de representación y razonamiento sobre determinados tipos de entidades para un conjunto específico de propósitos y eventos de importancia ecológica. Son de dominio específico, es decir, representan solo un pequeño subconjunto de cosas y eventos; de tarea específica, o sea que funcionan para resolver un conjunto limitado de problemas y están encapsulados, porque operan con un alto grado de independencia de otros sistemas cognitivos. De igual manera, los SCC aparecen tempranamente en la ontogenia y filogenia humana, pueden ser observados en todos los infantes de nuestra especie, y guían y moldean la vida mental de los adultos, ya que contribuyen al posterior funcionamiento y complejidad cognitiva en la especie (Gauvrit et al., 2017; Gelman, 2015).

\section{Definición de los cinco SCC para Spelke y colaboradores}

Spelke y Kinzler (2007) encuentran que los sistemas centrales del conocimiento son cinco y pueden encargarse de conocimientos relacionados con la representación de objetos, 
la representación de agentes y sus acciones, el sistema del número, la representación de la geometría y el entorno y, por último, la identificación de grupos sociales.

La representación de objetos se centra en los principios de cohesión o integración, continuidad y contacto (Aguiar \& Baillargeon, 1999; Leslie \& Keeble, 1987; Spelke, 1990), además de facilitar la percepción y comprensión de límites de los objetos, las formas, los movimientos, la predicción de la temporalidad y lugar de reposo del movimiento (Cadieu et al., 2014).

Un segundo sistema central estaría implicado con la representación de los agentes y sus acciones, las cuales son guiadas por la consecución de objetivos específicos y realizadas gracias a la obtención de los medios para alcanzarlas; lo anterior suele estar relacionado con las acciones y experiencias propias (Skerry, Carey, \& Spelke, 2013; Woodward \& Gerson, 2014).

El tercero es el sistema del número, cuyas representaciones son imprecisas, lo cual aumenta linealmente, además son abstractas, es decir, se aplican a diversas entidades encontradas a través de varias modalidades sensoriales. Las representaciones numéricas pueden ser comparadas y combinadas por operaciones de adición y sustracción (Gelman, 2015; Hyde, Khanum, \& Spelke, 2014).

El cuarto sistema media la representación de la geometría del entorno, las propiedades espaciales $\mathrm{y}$, en algunos casos, las propiedades geométricas de objetos móviles (Lee, Sovrano \& Spelke, 2012; Lee, Spelke \& Vallortigara, 2012; Spelke et al., 2010). El quinto y último sistema permitiría la identificación y razonamiento de grupos sociales $\mathrm{y}$ pares sociales potenciales, puesto que hay una tendencia a categorizarse a sí mismo y a otros, en grupos, y dicha agrupación lleva a la preferencia por los miembros del grupo interno (ingroup) vs. el grupo externo (outgroup) (Mulvey, Buchheister, \& McGrath, 2016; Shutts, Roben, \& Spelke, 2013).

\section{Variables individuales y SCC}

Aunque se ha discutido que el desarrollo cognitivo se fundamenta sobre una base genética transversal a la especie humana en un número de sistemas específicos que representan objetos, espacio y el número, los cuales surgen en la infancia y persisten durante toda la vida (Spelke, 2005), diferentes postulados consideran que el desarrollo cognitivo temprano podría diferenciarse entre niñas y niños. Por ejemplo, se ha señalado que los niños son más proclives que las niñas a desarrollar el conocimiento requerido por las matemáticas y las ciencias, porque los niños desde pequeños están más interesados por los objetos y sus interacciones, mientras que las niñas desde pequeñas se orientan por lo social (Baron-Cohen, 2003; Browne, 2002).

$\mathrm{Tal}$ como se ha planteado previamente, los SCC son la base de la construcción del conocimiento, por lo cual un ambiente adecuado y saludable permitirían que individualmente se logre un mayor potencial en cada uno de los sistemas (Gelman, 2015). De este modo, se reconoce que junto a la interacción ambiental como parte del inter-juego del desarrollo cognitivo desde etapas tempranas, también pueden participar diversas variables individuales y sociodemográficas para lograr que los SCC interactúen de manera integral.

Esta investigación pretende entregar información relacionada con los niveles de integración y ciertas características sociodemográficas, tomándolas desde su efecto temporal y no definitivo en el desarrollo infantil (Fitzpatrick, McKinnon, Blair, \& Willoughby, 2014; Wagmiller, 2015). A continuación, se presentan los resultados obtenidos en la búsqueda de una posible relación entre los SCC, la edad, el sexo y el estrato socioeconómico (ESE) y un índice general de desempeño, calculado con base en el desempeño particular en cada SCC, en niños y niñas entre 37 y 72 meses de edad. 


\section{Método}

\section{Participantes}

La muestra fue de 328 participantes (164 niñas; 164 niños) con un rango de edad entre los 37 y 72 meses $(M=53.22$ meses; $D E=$ 10.07), habitantes de la región sabana centro de Cundinamarca y de Boyacá (Colombia). En la selección, se tuvo en cuenta la muestra y los rangos de edad asignados a tres grupos específicos (de 37 a 48 meses, de 49 a 60 meses y de 61 a 72 meses), definidos en el programa de investigación "Por un inicio parejo de la vida" (GiraldoHuertas, Cano \& Pulido-Álvarez, 2017). La muestra se asignó de manera proporcional a la cantidad de población menor de seis años en cada grupo de edad y por cada municipio participante (Tabla 1). En cada región, se seleccionaron bloques cartográficos urbanos y dentro de estos se construyó un marco de menores de seis años para incluirlos a todos, y luego seleccionar uno solo por hogar. Se utilizó el método de muestreo aleatorio simple sin reemplazamiento para la selección de los bloques. La muestra rural se restringió a los centros poblados, y se asignó proporcionalmente a la población reportada según el Departamento Administrativo Nacional de Estadística de Colombia (DANE). La muestra se calculó para ser representativa por región (conjunto de municipios del departamento).

\section{Tabla 1}

Características de las niñas y los niños participantes en función de sexo y grupo de edad

\begin{tabular}{|c|c|c|c|}
\hline Categoría & $n(\%)$ & $\begin{array}{l}\text { Edad promedio } \\
\text { (Meses) }\end{array}$ & $D E$ \\
\hline \multicolumn{4}{|l|}{ Sexo } \\
\hline Total Niñas & $164(50)$ & 53.03 & 10 \\
\hline Total Niños & $164(50)$ & 53.41 & 10.16 \\
\hline \multicolumn{4}{|l|}{ Grupo Etáreo } \\
\hline Niños y niñas de 37 a 48 meses & $119(36.2)$ & 42.39 & 3.58 \\
\hline Niños y niñas de 49 a 60 meses & $115(35.1)$ & 54.05 & 3.59 \\
\hline Niños y niñas de 61 a 72 meses & $94(28.7)$ & 65.92 & 3.37 \\
\hline Total de la muestra & $328(100)$ & 53.22 & 10.07 \\
\hline
\end{tabular}

\section{Instrumentos}

Cada tarea se diseñó teniendo en cuenta la descripción del paso de las habilidades cognitivas tempranas de cada SCC hasta la edad adulta
(Spelke \& Kinzler, 2007). En los ajustes de la descripción que hacen los autores, se utilizaron los diseños de diversos estudios infantiles en cada SCC y las consecuentes tareas para observar y calcular el desempeño individual de cada participante.

\section{Situación de reconocimiento funcional del objeto}

Esta tarea consistía en la ubicación de diferentes objetos en espuma que previo reconocimiento por parte del niño(a), eran insertados por el mismo participante en sobres marcados con el nombre y una figura del mismo material perteneciente a la misma categoría en los que estos estarían presentes (Kingo \& Krøjgaard, 2011). Las categorías fueron: Taller, Granja, Casa, Viaje y Colegio. La consigna verbal para el participante fue la siguiente: "Vamos a jugar. Yo tengo cinco amigos que quieren 1) ir a su casa, 2) ir al colegio, 3) ayudar en el taller, 4) estar en la granja y 5) ir de viaje [a medida que se nombra cada lugar, se va pasando el respectivo sobre con el nombre de cada lugar] y lo que vamos a hacer es mirar cuál de todas estas cosas [se señalan las figuras identificadas] le va a servir a cada niño en cada lugar. Las puedes enviar cómo tú quieras. Solo necesito que las tomes y las metas en cada bolsa para cada niño".

\section{Tareas del número}

Esta tarea constó de tres fases, una de equivalencia, una de escritura y finalmente una de lectura. Primero, se trabajó con dos presentaciones de tres números, mediante la distribución de cubos de madera en dos sobres por número, uno con una distribución convencional de cubos (unidades de 10 y de 1 correspondientes a cada dígito del numeral escrito, por ejemplo, $21=$ dos cubos de 10 y un cubo de 1) y una presentación no convencional (unidades de 10 y de 1 no correspondientes a cada dígito del numeral escrito, por ejemplo, $21=$ un cubo de 10 y once cubos de 1). Se vaciaron los sobres marcados con el número correspondiente frente al niño (la marca es para facilidad del 
entrevistador; el niño no recibe los sobres) y se esperaba que este dijera si era mayor, menor o igual, con la expectativa de que identificara la equivalencia (Barth, Kanwisher, \& Spelke, 2003; Barth, La Mont, Lipton, \& Spelke, 2005). En una segunda fase, se le dictaban los tres números al niño $(12,21$ y 24) uno a uno, a medida que este los escribía en una hoja blanca. La fase 3 constaba de la presentación de los tres números impresos en una hoja blanca y la petición de la lectura por parte del niño del número presentado.

\section{Situación de ubicación espacial-geometría}

En esta tarea, un osito quería esconder su balón, para esto se disponía de un mapa donde con la marcación de una estrella se identificaba el lugar donde este balón debía estar escondido, con lo cual se esperaba que el niño ubicara una figura en espuma de un balón sobre una maqueta idéntica a la imagen del mapa. En todo momento, el niño tiene visible el mapa, el cual tiene la misma dirección que la maqueta para las tres primeras aplicaciones. En la cuarta aplicación, el mapa requiere que el niño identifique que al coincidir la ubicación del balón y la estrella en su presentación, pero con las marcas espaciales en sentido contrario, se debe invertir el mapa o ubicar en sentido contrario la localización del balón para que corresponda con la estrella (Spelke et al., 2010).

\section{Situación de igualdad entre agentes}

Se presentaron dos cajas pertenecientes a dos personajes: uno tenía tres y otro dos pegatinas (stickers) de las llamadas "caritas felices" dentro de la caja; inicialmente, se entregaban cuatro pegatinas al niño(a) para que repartiera entre ambos personajes, sin saber que poseían una cantidad diferente; después se abrían las cajas y tras el conocimiento de la cantidad de lo que poseían, se le entregaban de nuevo cuatro pegatinas para repartir $y$, por último, se le pedía al niño(a) que repartiera los últimos tres pegatinas dentro de las cajas de los personajes, esperando que la repartición terminase en igualdad (Schmidt \& Sommerville, 2011).

\section{Situación nosotros-ellos}

Se entregaron al niño(a) 10 pegatinas de caritas felices para que repartiera en dos sobres, uno para sus padres y otro para un niño desconocido (Shutts, Banaji, \& Spelke, 2010). Esta tarea se deriva del juego del Dictador en economía experimental y aplicado a niños (Benenson, Pascoe, \& Radmore, 2007).

El desempeño en cada tarea se calificó teniendo en cuenta el resultado o la respuesta de cada actividad propuesta (Tabla 2).

\section{Tabla 2}

Forma de calificación de las 5 tareas y obtención del indice general de desempeño sociocognitivo

\begin{tabular}{|c|c|}
\hline Tarea & Descripción de calificación \\
\hline $\begin{array}{l}\text { Reconocimiento funcional de } \\
\text { objeto }\end{array}$ & $\begin{array}{l}\text { Se calificó de acuerdo a la inserción de cada objeto en el sobre } \\
\text { correspondiente al contexto en el que se encontraria, asi al tener en cuenta } \\
\text { que se presentaron cinco escenarios con cinco estimulos relacionados, se } \\
\text { contaron el número de aciertos del participante, sobre el número total de } \\
\text { posibles aciertos, es decir, } 25 \text {. }\end{array}$ \\
\hline Tarea del número & $\begin{array}{l}\text { El criterio de calificación se basó en la frecuencia de aciertos y } \\
\text { desaciertos en cada uno de los números que compone esta tarea (escritura, } \\
\text { lectura y equivalencia), se asignaron valores de una escala de } 2 \text { a } 18 \text { ( } 2 \\
\text { para la mayor frecuencia en acierto y } 18 \text { para la menor frecuencia en } \\
\text { aciertos) y de }-1 \text { a - }-9 \text { para los desaciertos: - } 1 \text { para la menor frecuencia en } \\
\text { aciertos y - } 9 \text { para la mayor frecuencia en aciertos. }\end{array}$ \\
\hline $\begin{array}{l}\text { Tarea de ubicación geométrica- } \\
\text { espacial }\end{array}$ & $\begin{array}{l}\text { Se tuvo en cuenta el registro de los cuadrantes donde tuvo contacto el } \\
\text { sticker. Este registro se hizo por cada mapa y se estimó de } 5 \text { a } 1 ; 5 \text { para el } \\
\text { puntaje más alto y } 1 \text { para el puntaje más bajo, correspondientes con el } \\
\text { cuadrante más cercano o exacto en la posición target y al más distante. } \\
\text { Para conocer el desempeño de cada uno de los niños en los mapas, se } \\
\text { sumaron los valores obtenidos por los tres mapas sobre } 15 \\
(+3 \text { MAPAS } / 15) ; 15 \text { porque el puntaje ideal más alto por cada uno de los } \\
\text { mapas era } 5 \text {. }\end{array}$ \\
\hline $\begin{array}{l}\text { Tarea de agentes-faimess y } \\
\text { tarea de nosotros-ellos }\end{array}$ & $\begin{array}{l}\text { Se calificaron de acuerdo con la distribución y compensación que hacian } \\
\text { de las entregas a cada uno de los personajes de la situación de desigualdad } \\
\text { (Juanita y Pepita) y de la distribución equitativa entre familia y extraño de } \\
\text { la situación nosotros-ellos. }\end{array}$ \\
\hline Indice general de desempeño & $\begin{array}{l}\text { El cálculo acumulado de los desempeños en cada tarea permitió asignar } \\
\text { un indice de desempeño general para cada participante al tazar el } 100 \% \\
\text { del desempeño general a partir de la suma de cada puntaje en cada tarea y } \\
\text { la proporción obtenida de cada participante bajo el resultado esperado sin } \\
\text { errores, es el indice individual resultante. }\end{array}$ \\
\hline
\end{tabular}

\section{Procedimiento}

Tras el contacto telefónico con los cuidadores, se realizó una sesión mediante visita domiciliaria, para firmar el consentimiento de participación en el estudio por parte de los acudientes del niño participante. El consentimiento contaba con la inclusión de la normatividad colombiana donde se resalta que los derechos de los niños y las niñas prevalecen sobre los demás, en especial, si existe conflicto entre sus derechos fundamentales con los de cualquier otra persona (Ley 1098 de 2006, Código de la Infancia y la Adolescencia). 
Una vez firmado el consentimiento, el niño y el evaluador iniciaban con un tiempo de juego libre. Posteriormente, se aplicaron en orden las cinco tareas, esto es, primero la situación de reconocimiento funcional del objeto, seguido de las tareas del número, situación de ubicación espacial-geometría, agentes fairness y la situación nosotros-ellos, respectivamente, las cuales se calificaron subsiguientemente de acuerdo con los criterios descritos antes. El protocolo de evaluación de desarrollo cognitivo estableció un tiempo aproximado de evaluación entre 45 y 90 minutos.

\section{Análisis de datos}

Los desempeños se calificaron con una base proporcional de acierto y error que permite un dato por tarea y un índice general acumulado para cada participante. Este índice de desempeño general, aplicado de manera conjunta con un instrumento de monitoreo del desarrollo, basado en la Tabla de Desarrollo Psicomotor (Servicio de Salud Vasco, 1991) derivada del test Denver II que se presenta en el Estudio Haizea-Llevant (Fernández Álvarez, 1989; Fernández-Álvarez, Fernández-Matamoros, Fuentes, \& Rueda, 1991; Iceta \& Yoldi, 2002), a una muestra de 1 177 participantes (Giraldo-Huertas et al., 2017), obtuvo un alto coeficiente de confiabilidad (alfa de Cronbach 0.92). Este resultado previo permite denominar los resultados del índice de desempeño general cómo indice general de desarrollo sociocognitivo, para evitar confusiones con los desempeños en cada una de las tareas.

Los datos de este índice junto a las otras variables de interés, fueron inicialmente analizados con la prueba de normalidad Kolmogorov-Smirnov y, al no identificar una distribución normalizada, se usaron pruebas no paramétricas como la prueba $U$ de MannWhitney para identificar diferencias entre edad, sexo y ESE, y correlaciones no paramétricas de Spearman para hallar relaciones entre los SCC.

\section{Resultados}

Con el fin de analizar el efecto de la edad sobre los índices de desempeño en las cinco tareas y el índice general de desempeño sociocognitivo, utilizando para esto tres grupos de edad (de 37 a 48 meses, de 49 a 60 meses y de 61 a 72 meses), se aplicó una prueba Kruskal-Wallis, encontrando diferencias significativas en el índice general de desempeño sociocognitivo en función de la edad de los participantes $\left(\chi^{2}=85.7, p<0.001\right)$. Una corrección de Bonferroni se aplica para reportar el nivel de significancia a partir de 0.016. Por tanto, se mantiene el efecto de la edad sobre el desempeño general. En las comparaciones con la prueba Bonferroni (post hoc), el índice de desempeño general del rango de edad de 37 a 48 meses fue significativamente diferente al obtenido por el rango de 49 a 60 meses $(p<$ $0.001, r=0.33)$ y al obtenido por el rango de 61 a 72 meses $(p<0.001, r=0.6)$; de igual manera, el desempeño del rango de 49 a 60 meses fue significativamente diferente al del rango de 61 a 72 meses $(p<0.001, r=0.38)$, que se muestra en la Figura 1.

\section{Figura 1}

Comparación en los tres rangos de edad en las medias de desempeño obtenido en el índice de desarrollo sociocognitivo

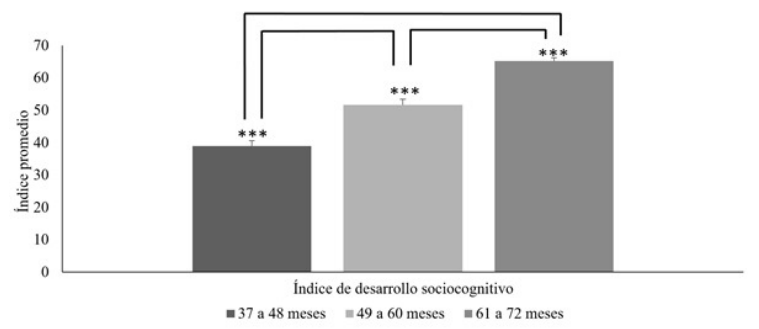

En segunda instancia, se encuentran diferencias significativas en el Kruskal-Wallis de manera particular en las tareas de reconocimiento funcional del objeto $\left(\chi^{2}=54.2\right.$, $p<0.001$ ), encontrando en el post-hoc que el desempeño del rango de edad de 37 a 48 meses fue significativamente diferente a la media obtenida por el rango de 49 a 60 meses $(p<$ $0.001, r=0.28$ ) y a la obtenida por el rango de 
61 a 72 meses $(p<0.001, r=0.48)$; asimismo, el desempeño del rango de 49 a 60 meses fue significativamente diferente al de 61 a 72 meses $(p<0.001, r=0.27)$, como se muestra en la Figura 2.

\section{Figura 2}

Comparación en los tres rangos de edad en las medias del desempeño obtenido en los índices reconocimiento funcional de objetos

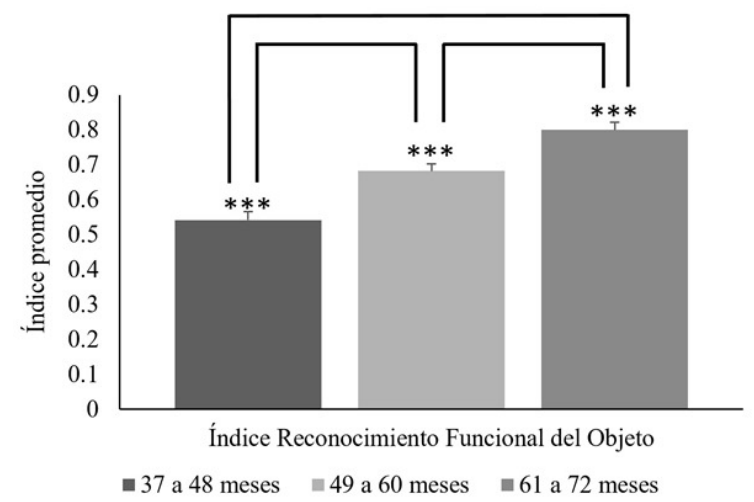

Adicionalmente, se encontraron diferencias significativas en las tareas del número $\left(\chi^{2}=\right.$ 85,735, p $<0.001$ ), el post-hoc, indicó que el desempeño del rango de edad de 37 a 48 meses fue significativamente diferente a la media obtenida por el rango de 49 a 60 meses $(p<$ $0.001, r=0.24)$ y a la obtenida por el rango de 61 a 72 meses $(p<0.001, r=0.62)$; de igual manera, el desempeño del rango de 49 a 60 meses fue significativamente diferente al del rango de 61 a 72 meses $(p<0.001, r=0.41)$, como se observa en la Figura 3.

\section{Figura 3}

Comparación en los tres rangos de edad en las medias de desempeño promedio obtenido en los indices de las tareas del número

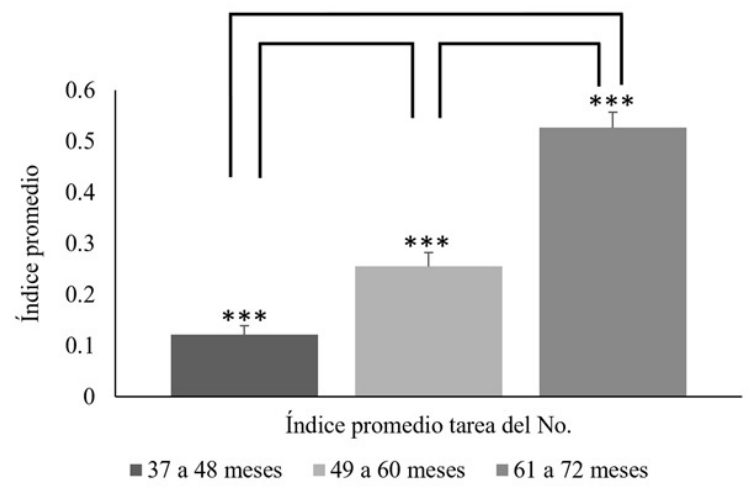

Finalmente, sobre las diferencias analizadas en el Kruskal-Wallis en las tareas de Ubicación espacial $\left(\chi^{2}=8.258, p<0.016\right)$, el posthoc permitió evidenciar diferencias significativas entre el desempeño del rango de edad de 37 a 48 meses y el rango de 61 a 72 meses $(p=0.015, r$ $=0.2$ ), tal como se presenta en la Figura 4 .

\section{Figura 4}

Comparación en los tres rangos de edad en las medias de desempeño promedio obtenido en los indices de tareas de ubicación espacial

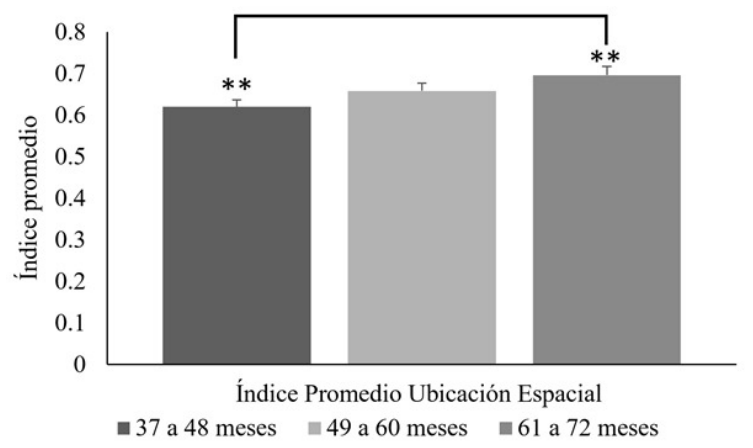

Con el fin de analizar el efecto de la variable sexo sobre el desempeño en las tareas y en el índice general de desempeño sociocognitivo, se aplicó una prueba Mann-Whitney, en la que se encontró que el desempeño general de desarrollo sociocognitivo, al igual que las cinco tareas particulares de SCC, no se ve afectados significativamente por el sexo de los participantes. 
El análisis de correlaciones de Spearman realizado entre las cinco tareas cognitivas y el índice de desempeño general, mostró una correlación significativa entre el índice de desarrollo sociocognitivo y los sistemas de reconocimiento funcional del objeto, tareas del número y tareas de ubicación espacial; asimismo, hubo una correlación significativa entre agentes faimess y nosotros vs. ellos, entre el reconocimiento funcional del objeto y las tareas del número y, por último, entre agentes fairness y las tareas de ubicación espacial (Tabla 3).

\section{Tabla 3}

Coeficiente de correlación (rho de Spearman) entre el ranking obtenido en los 5 grupos de tareas y el indice de desarrollo sociocognitivo $(N=328)$

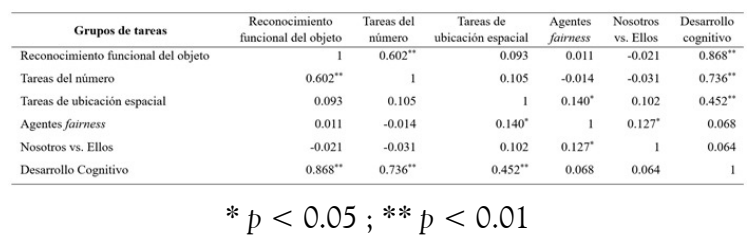

En cuanto al ESE de los participantes y las variables analizadas, al aplicar una prueba Kruskal-Wallis, para considerar el nivel de estrato socioeconómico sobre los resultados del sexo femenino, no se encontró efecto sobre el índice de desempeño general $\left(\chi^{2}=7.759, p>0.05\right)$ o las cinco tareas de SCC. De igual manera, al analizar el efecto del estrato socioeconómico sobre los resultados del sexo masculino, no se encontró efecto sobre el índice de desempeño general $\left(\chi^{2}=1.408, p>0.05\right)$ o las cinco tareas de SCC. Del mismo modo, la aplicación del Kruskal-Wallis permitió reconocer que no hubo efecto del nivel socioeconómico sobre ninguno de los tres grupos de edad.

\section{Discusión}

La relación ente las variables estudiadas y los desempeños de los niños participantes en las diversas tareas de los SCC permiten reconocer varias características importantes que no se han descrito previamente y que pretenden mantener el interés en una mayor evidencia en cuanto a la integración de los sistemas y los efectos de variables tanto ambientales como genéticas, durante el desarrollo individual. En los presentes resultados, la edad acompaña los cambios en los SCC e indica que, al parecer, después de los tres primeros años de vida, tales cambios son progresivos e incrementan en función del aumento de la edad, tal y como se reporta para otras habilidades cognitivas a nivel individual y de la especie (Benítez-Burraco \& Uriagereka, 2015). Este hallazgo implica que la base ontogenética de los SCC puede llegar a considerarse, bajo mayor exploración de variables ambientales y ontogenéticas de la especie, como generalizable para cada individuo, al igual que otros conceptos y constructos relacionados con el desarrollo cognitivo en general (Carey, 2009; Gelman, 2003; Mandler, 2004).

En cada SCC, los efectos de la edad se mantienen, lo cual permite reconocer los hallazgos reportados en otras investigaciones sobre el conocimiento de los objetos (Mareschal, French, \& Quinn, 2000), el conocimiento numérico y aritmético (LeFevre et al., 2009; LeFevre et al., 2010; Levine, Suriyakham, Rowe, Huttenlocher, \& Gunderson, 2010; Lukie, Montazer-Hojat, \& Holroyd, 2014; Melhuish et al., 2008; Mix, Sandhofer, Moore, \& Russell, 2012) y el conocimiento geométrico y el uso de mapas (DeLoache, 2000; Huttenlocher, Vasilyeva, Newcombe, \& Duffy, 2008; Uttal, Schreiber, \& DeLoache, 1995). En contraste con otros estudios (Schmidt \& Sommerville, 2011), las tareas relacionadas con la representación de los agentes y sus acciones dirigidas a metas en la tarea agentes-fairness y el procesamiento de información social en la situación nosotros-ellos, no fueron afectadas por la variable edad.

¿Por qué no se mantienen los efectos de la edad en lo social? Estudios recientes demuestran una diferencia notable antes y después los tres años de edad en las habilidades de detección de la desigualdad y la distribución equitativa de recursos (Schmidt, Butler, Heinz, \& Tomasello, 2016). Tales hallazgos indican preferencias igualitarias y orientadas por expectativas de equidad entre pares antes de los tres años de edad, lo cual indica un cierto interés por el bienestar de otros (Brownell, Svetlova, 
\& Nichols, 2009; Dunfield \& Kuhlmeier, 2013; Schmidt \& Sommerville, 2011; Schmidt, Rakoczy, \& Tomasello, 2012; Sloane, Baillargeon, \& Premack, 2012; Sommerville, Schmidt, Yun, \& Burns, 2013; Svetlova, Nichols, \& Brownell, 2010). Sin embargo, solo después de los tres años es posible encontrar una noción de equidad más generalizable y aplicada como principio en diversas situaciones de distribución de recursos (Blake \& McAuliffe, 2011; Fehr, Bernhard, \& Rockenbach, 2008; Paulus, 2015; Shaw \& Olson, 2012).

Por tanto, los resultados en esta investigación para los sistemas de procesamiento de información social pueden estar relacionados con que la exigencia de imparcialidad entre agentes en un contexto donde no se reconoce el origen de los recursos, ni las condiciones particulares en situaciones distributivas, lo cual podría limitar el uso de información de contexto que resulta relevante para decisiones de este tipo en edades tan tempranas y fundamentales en la distribución de recursos materiales (Elenbaas, Rizzo, Cooley, \& Killen, 2016). Sin embargo, las correlaciones indican una asociación entre las dos tareas sociales (agentes-fairness y nosotros vs. ellos) y una de ellas (agentes faimess) con las tareas de ubicación espacial. Se debe mantener la exploración de este vínculo en futuras investigaciones con mayores controles poblacionales e instrumentales que permitan comprender los vínculos entre el conocimiento social y habilidades cognitivas generales.

A diferencia de la edad, el sexo y el ESE no muestran efectos relevantes en los desempeños de los niños participantes. ¿Por qué persisten entonces en otros estudios sobre habilidades cognitivas y el desarrollo en general el reporte de efectos de estas variables? No se puede ofrecer una respuesta definitiva, pero la sensibilidad de las tareas, la educación y el contexto inmaterial en los ambientes de aprendizaje pueden indicar una respuesta provisional, específicamente para el desarrollo de habilidades sociales (Barbu, Cabanes, \& Le Maner-Idrissi, 2011; Walker, Irving, \& Berthelsen, 2002) y junto a los cambios que se generarían después de la pubertad (Barbu et al., 2011; Ingalhalikar et al., 2014). De hecho, la modalidad de las tareas aplicadas, que en su condición de instrumentos no verbales implica recurrir a observaciones comportamentales, han mostrado, desde hace décadas, hallazgos no significativos para las variables mencionadas (Eisenberg \& Lennon, 1983).

La relevancia de la educación y el contexto se reafirman junto a una de las características más importantes de los SCC, bajo el supuesto de que el desarrollo de los dominios en los SCC responde principalmente a características propias de la especie humana y el ESE, como variable de agrupación o clasificación de acuerdo con las características sociales y económicas, principalmente de la vivienda en zonas urbanas (Gouin et al., 2015), no tendría por qué afectar el desempeño asociado a los SCC. Los hallazgos guardan coherencia con los planteamientos recientes que reconocen que el ESE no afecta el desarrollo, a diferencia de escenarios de aprendizaje y entornos de condiciones inadecuadas, como el estrés (Diamond, 2013).

En conclusión, los resultados obtenidos permiten confirmar que además de una alta correlación entre ellos, los SCC reflejan una alta sensibilidad a variables relacionadas con los cambios ontogenéticos de la especie (Spelke, 1990; Spelke, 2000, 2005; Spelke \& Kinzler, 2007; Spelke et al., 2010) y en el caso de la población y las tareas aplicadas, no dependen de igual manera de factores contextuales como el ESE o las prácticas orientadas por el sexo en edad preescolar, aportando evidencia para considerar que dicho funcionamiento es transversal en la especie humana. Por tanto, es posible que la atención temprana de los SCC en escenarios educativos y entornos familiares, evite las desigualdades que se presentan en las diferencias sociales posteriores y bajo dinámicas actuales de desigualdad (Diamond, 2013; Richter et al., 2016).

Finalmente, se recomienda que en próximos estudios se tengan en cuenta variables que permitan evidenciar el posible efecto de la interacción de los niños con su entorno sobre el desarrollo sociocognitivo, como la participación en escenarios educativos y las actividades en el hogar, las cuales han demostrado un efecto 
inmediato y posterior en el desarrollo individual (Britto et al., 2016). Los SCC se enmarcan dentro de conceptos fundamentales y formales en la base de la cognición humana y hacen posible la consideración de oportunidades, para que los individuos alcancen el mayor potencial en su desarrollo y complejidad cognitiva antes de aparentes hitos de decadencia cognitiva en la especie (Gauvrit et al., 2017).

\section{Referencias}

Aguiar, A., \& Baillargeon, R. (1999). Perseveration and problem solving in infancy. Advances in Child Development and Behavior, 27, 135-180. https://doi.org/10.10 16/S0065-2407(08)60138-X

Barbu, S., Cabanes, G., \& Le Maner-Idrissi, G. (2011). Boys and girls on the playground: Sex differences in social development are not stable across early childhood. PLoS ONE, 6(1), e16407. https://doi.org/10.137 1/journal.pone.0016407

Barner, D., \& Baron, A. (Eds.) (2016). Core knowledge and conceptual change. Oxford: Oxford University Press.

Baron-Cohen, S. (2003). The essential difference: The truth about the male and female brain. New York: Basic Books.

Barth, H., Kanwisher, N., \& Spelke, E. (2003). The construction of large number representations in adults. Cognition, 86(3), 201-221. Recuperado de https://wesfiles.we sleyan.edu/home/hbarth/web/PublicWebFi les/BarthEtAl_Cognition03.pdf

Barth, H., La Mont, K., Lipton, J., \& Spelke, E. S. (2005). Abstract number and arithmetic in preschool children. Proceedings of the National Academy of Sciences of the United States of America, 102(39), 14116-14121. Recuperado de https://wesfiles.wesleyan.ed u/home/hbarth/web/PublicWebFiles/Barth EtAl_PNAS05.pdf

Benenson, J., Pascoe, J. \& Radmore, N. (2007). Children's altruistic behavior in the dictator game. Evolution and Human Behavior, 28(3),
168-175. https://doi.org/10.1016/j.evolhum behav.2006.10.003

Benítez-Burraco, A., \& Uriagereka, J. (2015). The immune syntax revisited: Opening new windows on language evolution. Frontiers in Molecular Neuroscience, 8, 84. https://doi.or $\mathrm{g} / 10.3389 /$ fnmol.2015.00084

Blake, P. R., \& McAuliffe, K. (2011). "I had so much it didn't seem fair": Eight-yearolds reject two forms of inequity. Cognition, 120 (2), 215-224. https://doi.org/10.1016/j. cognition.2011.04.006

Britto, P. R., Lye, S. J., Proulx, K., Yousafzai, A. K., Matthews, S. G., Vaivada, T., ... Early Childhood Development Interventions Review Group (2016). Nurturing care: promoting early childhood development. Advancing Early Chilhood Development, 389(10064), 91-102. https://doi.org/10.101 6/S0140-6736(16)31390-3

Browne, K. R. (2002). Biology at work: Rethinking sexual equality. Piscataway, NJ: Rutgers University Press.

Brownell, C. A., Svetlova, M., \& Nichols, S. (2009). To share or not to share: When do toddlers respond to another's needs? Infancy, 14(1), 117-130. https://doi.org/10. 1080/15250000802569868

Carey, S. (2009). The origin of concepts. Oxford: Oxford University Press.

Cadieu, C., Hong, H., Yamins, D., Pinto, N., Ardila, D., Solomon, E., ... DiCarlo, J. (2014). Deep neural networks rival the representation of primate IT cortex for core visual object recognition. PLoS Computational Biology, 10(12), e1003963. https://doi.org/10.1371/journal. pcbi. 1003963

DeLoache, J. S. (2000). Dual representation and young children's use of scale models. Child Development, 71 (2), 329-338. https://doi.or $\mathrm{g} / 10.1111 / 1467-8624.00148$

Diamond, A. (2013). Executive functions. Annual Review of Psychology, 64, 135-168. https://doi.org/10.1146/annurevpsych-113011-143750

Dunfield, K. A., \& Kuhlmeier, V. A. (2013). Classifying prosocial behavior: 
Children's Responses to instrumental need, emotional distress, and material desire. Child Development, 84(5), 1766-1776. https ://doi.org/10.1111/cdev.12075

Eisenberg, N., \& Lennon, R. (1983). Sex differences in empathy and related capacities. Psychological Bulletin, 94(1), 100-131. https://doi.org/10.1037/0033-290 9.94

Elenbaas, L., Rizzo, M. T., Cooley, S., \& Killen, M. (2016). Rectifying social inequalities in a resource allocation task. Cognition, 155, 176-187. https://doi.org/10.1016/j.cognitio n.2016.07.002

Fehr, E., Bernhard, H., \& Rockenbach, B. (2008). Egalitarianism in young children. Nature, 454(7208), 1079-1083. Recuperado de http://www.nature.com/nature/journal/ v454/n7208/suppinfo/nature07155_S1.ht $\mathrm{ml}$

Fernández Álvarez E. (1989). El desarrollo psicomotor de 1.702 niños de 0 a 24 meses. [The psyco-motor development of 1702 children 0 to 24 monthsold] (Unpublished doctoral Thesis)., Universidad de Barcelona, Barcelona.

Fernández-Álvarez, E., Fernández-Matamoros, I., Fuentes, J., \& Rueda, J. (1991). Escala Haizea-Llevant. Vitoria: Servicio Central de Publicaciones. Gobierno Vasco.

Fitzpatrick, C., McKinnon, R., Blair, C., \& Willoughby, M. (2014). Do preschool executive function skills explain the school readiness gap between advantaged and disadvantaged children?. Learning and Instruction, 30, 25-31. https://doi.org/10.10 16/j.learninstruc.2013.11.003

Fodor, J. A. (1983). Modularity of mind. Cambridge, MA: MIT Press.

Gauvrit, N., Zenil, H., Soler-Toscano, F., Delahaye, J. -P. \& Brugger, P. (2017). Human behavioral complexity peaks at age 25. PLoS Computational Biology, 13(4), e1005408. https://doi.org/10.1371/journal. pcbi. 1005408

Gelman, R. (2015). Learning in core and non-core number domains. Developmental
Review, 38, 185-200. https://doi.org/10.101 6/j.dr.2015.07.0100273-2297

Gelman, S. A. (2003). The essential child: Origins of essentialism in everyday thought. Nueva York: Oxford University Press.

Giraldo-Huertas, J., Cano, L., \& Pulido-Álvarez, A. (2017). Desarrollo socio-cognitivo en la primera infancia: los retos por cumplir en salud pública en la zona Sabana Centro y Boyacá. Revista de Salud Pública, 19(4), 484-490. https://doi.org/10.15446/rsap.v19 n4.51787

Gouin, M., Flamant, C., Gascoin, G., Rouger, V., Florin, A., Guimard, P., ... Hanf, M. (2015). The association of urbanicity with cognitive development at five years of age in preterm children. PLoS ONE, 10(7), e0131749. htt ps://doi.org/10.1371/journal.pone.0131749

Hyde, D. C., Khanum, S., \& Spelke, E. S. (2014). Brief non-symbolic, approximate number practice enhances subsequent exact symbolic arithmetic in children. Cognition, 131(1), 92-107. https://doi.org/1 0.1016/j.cognition.2013.12.007

Huttenlocher, J., Vasilyeva, M., Newcombe, N., \& Duffy, S. (2008). Developing symbolic capacity one step at a time. Cognition, 106(1), 1-12. https://doi.org/10.1016/j.cog nition.2006.12.006

Iceta, A., \& Yoldi, M. (2002). Desarrollo psicomotor del nin\#o y su valoracio\#n en atencio\#n primaria. ANALES del Sistema Sanitario de Navarra, 25(Supl. 2), 35-43. Recuperado de https://pdfs.semanticscholar.org/c26b/d da557fcc4260a6dcdec03872d2a66f6f22a.p df

Ingalhalikar, M., Smith, A., Parker, D., Satterthwaite, T. D., Elliott, M. A., Ruparel, K., ... Verma, R. (2014). Sex differences in the structural connectome of the human brain. Proceedings of the National Academy of Sciences, 111 (2), 823-828. https://doi.org/1 0.1073/pnas.1316909110

Karmiloff-Smith, A., Crespo, J. C. G., \& Bernardos, M. N. (1994). Más allá de la modularidad: la ciencia cognitiva desde la perspectiva del desarrollo: Madrid: Alianza. 
Kingo, O. S., \& Krøjgaard, P. (2011). Object manipulation facilitates kind-based object individuation of shape-similar objects. Cognitive Development, 26(2), 87-103. https ://doi.org/10.1016/j.cogdev.2010.08.009

Lee, S. A., Sovrano, V. A., \& Spelke, E. S. (2012). Navigation as a source of geometric knowledge: Young children's use of length, angle, distance, and direction in a reorientation task. Cognition, 123(1), 144-161. https://doi.org/10.1016/j.cognitio n.2011.12.015

Lee, S. A., Spelke, E. S., \& Vallortigara, G. (2012). Chicks, like children, spontaneously reorient by threedimensional environmental geometry, not by image matching. Biology Letters, 8(4), 492-494. https://doi.org/10.1098/rsbl.2012. 0067

LeFevre, J. -A., Fast, L., Skwarchuk, S. -L., Smith-Chant, B. L., Bisanz, J., Kamawar, D., \& Penner-Wilger, M. (2010). Pathways to mathematics: Longitudinal predictors of performance. Child Development, 81(6), 1753-1767. https://doi.org/10.1111/j.14678624.2010.01508

LeFevre, J. -A., Skwarchuk, S. -L., Smith-Chant, B. L., Fast, L., Kamawar, D., \& Bisanz, J. (2009). Home numeracy experiences and children's math performance in the early school years. Canadian Journal of Behavioural Science, 41 (2), 55-66. https://d oi.org/10.1037/a0014532

Leslie, A. M., \& Keeble, S. (1987). Do six-monthold infants perceive causality? Cognition, 25(3), 265-288. https://doi.org/10.1016/SO 010-0277(87)80006-9

Levine, S. C., Suriyakham, L. W., Rowe, M. L., Huttenlocher, J., \& Gunderson, E. A. (2010). What counts in the development of young children's number knowledge? Developmental Psychology, 46(5), 1309-1319. https://doi.org/10.1037/ a0019671

Lukie, C. N., Montazer-Hojat, S., \& Holroyd, C. B. (2014). Developmental changes in the reward positivity: An electrophysiological trajectory of reward processing. Developmental Cognitive Neuroscience, 9, 191-199. https://doi.org/10 .1016/j.dcn.2014.04.003

MacDonald, K., \& Hershberger, S. (2005). Theoretical issues in the study of evolution and development. Evolutionary Perspectives on Human Development, 2, 21-72. https://do i.org/10.4135/9781452233574.n2

Mandler, J. M. (2004). The foundations of mind: Origins of conceptual thought. Oxford: Oxford University Press.

Mareschal, D., French, R. M., \& Quinn, P. C. (2000). A connectionist account of asymmetric category learning in early infancy. Developmental Psychology, 36(5), 635-645. https://doi.org/10.1037/0012-164 9.36.5.635

Melhuish, E. C., Phan, M. B., Sylva, K., Sammons, P., Siraj-Blatchford, I., \& Taggart, B. (2008). Effects of the home learning environment and preschool center experience upon literacy and numeracy development in early primary school. Journal of Social Issues, 64(1), 95-114. https://doi.org/10.1111/j.1540-456 0.2008.00550.x

Mix, K. S., Sandhofer, C. M., Moore, J. A., \& Russell, C. (2012). Acquisition of the cardinal word principle: The role of input. Early Childhood Research Quarterly, 27(2), 274-283. https://doi.org/10.1016/j.ecresq.2 011.10 .003

Mulvey, K. L., Buchheister, K., \& McGrath, K. (2016). Evaluations of intergroup resource allocations: The role of theory of mind. Journal of Experimental Child Psychology, 142, 203-211. https://doi.org/10.1016/j.jecp .2015 .10 .002

Palacios, J. (1990). Psicología evolutiva: conceptos, enfoques, controversias y métodos. En J. Palacios, A. Marchesi \& C. Coll (Coords.), Desarrollo psicológico y educación (Vol. 1, pp. 23-80). Madrid: Alianza.

Paulus, P. B. (Ed.). (2015). Psychology of group influence (2.a ed., Vol. 22). Londres: Psychology Press Ltd. 
Richter, L. M., Daelmans, B., Lombardi, J., Heymann, J., Boo, F. L., Behrman, J. R., ... Darmstadt, G. L. (octubre, 2016). Investing in the foundation of sustainable development: Pathways to scale up for early childhood development. Lancet. https://doi .org/10.1016/S0140-6736(16)31698-1

Rips, L. J. (2017). Core cognition and its aftermath. Philosophical Topics, 45(1), 157-179. https://doi.org/10.5840/philtopics 20174519

Schmidt, M. F., Butler, L. P., Heinz, J., \& Tomasello, M. (2016). Young children see a single action and infer a social norm: Promiscuous normativity in 3-year-olds. Psychological Science, 27(10), 1360-1370. ht tps://doi.org/10.1177/0956797616661182

Schmidt, M. F., \& Sommerville, J. A. (2011). Fairness expectations and altruistic sharing in 15-month-old human infants. PLoS ONE, 6(10), e23223. https://doi.org/10.13 71/journal.pone.0023223

Schmidt, M. F. H., Rakoczy, H., \& Tomasello, M. (2012). Young children enforce social norms selectively depending on the violator's group affiliation. Cognition, 124(3), 325-333. https://doi.org/10.1016/j. cognition.2012.06.004

Servicio de Salud Vasco. (1991). Tabla de desarrollo psicomotor. En Estudio Haizea Llevant. Vitoria-Gasteiz: Servicio Central de Publicaciones. Gobierno Vasco.

Shutts, K., Roben, C. K. P., \& Spelke, E. S. (2013). Children's use of social categories in thinking about people and social relationships. Journal of Cognition and Development, 14(1), 35-62. https://doi.org/1 $0.1080 / 15248372.2011 .638686$

Shaw, A., \& Olson, K. R. (2012). Children discard a resource to avoid inequity. Journal of Experimental Psychology: General, 141 (2), 382-395. https://doi.org/10.1037/a0025907

Shutts, K., Banaji, M. R., \& Spelke, E. S. (2010). Social categories guide young children's preferences for novel objects. Developmental Science, 13(4), 599-610. https://doi.org/10. 1111/j.1467-7687.2009.00913.x
Skerry, A. E., Carey, S. E., \& Spelke, E. S. (2013). First-person action experience reveals sensitivity to action efficiency in prereaching infants. Proceedings of the National Academy of Sciences, 110(46), 18728-18733. https://doi.org/10.1073/pnas .1312322110

Sloane, S., Baillargeon, R., \& Premack, D. (2012). Do infants have a sense of fairness? Psychological Science, 23(2), 196-204. https: //doi.org/10.1177/0956797611422072

Sommerville, J. A., Schmidt, M. F. H., Yun, J. E., \& Burns, M. (2013). The development of fairness expectations and prosocial behavior in the second year of life. Infancy, 18(1), 40-66. https://doi.org/10.1111/j.153 2-7078.2012.00129.x

Spelke, E. S. (1990). Principles of object perception. Cognitive Science, 14(1), 29-56. https://doi.org/10.1207/s15516709c og1401_3

Spelke, E. S. (2000). Core knowledge. American Psychologist, 55(11), 1233-1243. doi: 10.1037/0003-066X.55.11.1233

Spelke, E. S. (2005). Sex differences in intrinsic aptitude for mathematics and science?: A critical review. American Psychologist, 60(9), 950-958. https://doi.org/10.1037/0003-066 X.60.9.950

Spelke, E. S., \& Kinzler, K. D. (2007). Core knowledge. Developmental Science, 10(1), 89-96. https://doi.org/10.1111/j.1467-7687 $.2007 .00569 . x$

Spelke, E., Lee, S. A., \& Izard, V. (2010). Beyond core knowledge: Natural geometry. Cognitive Science, 34(5), 863-884. https://d oi.org/10.1111/j.1551-6709.2010.01110.x

Svetlova, M., Nichols, S. R., \& Brownell, C. A. (2010). Toddlers' prosocial behavior: From instrumental to empathic to altruistic helping. Child Development, 81 (6), 1814-1827. https://doi.org/10.1111/j.14678624.2010.01512.x

Uttal, D., Schreiber, J. C., \& DeLoache, J. S. (1995). Waiting to use a symbol: The effects of delay on children's use of models. Child Development, 66(6), 1875-1889. https://doi. org/10.1111/j.1467-8624.1995.tb00971.x 
Wagmiller, R. L. (2015). The temporal dynamics of childhood economic deprivation and children's achievement. Child Development Perspectives, 9(3), 158-163. https://doi.org/1 $0.1111 /$ cdep. 12125

Walker, S., Irving, K., \& Berthelsen, D. (2002). Gender influences on preschool children's social problem-solving strategies. The Journal of Genetic Psychology, 163(2), 197-209. https://doi.org/10.1080/00221320 209598677

Woodward, A. L., \& Gerson, S. A. (2014). Mirroring and the development of action understanding. Philosophical Transactions of the Royal Society. B, 369(1644), 1-8. https:// doi.org/10.1098/rstb.2013.0181

\section{Notas}

* Artículo de investigación. Proyecto financiado por la Dirección General de Investigación, Universidad de la Sabana, Proyecto PSI-50-2015 de Convocatoria Interna de Menor Cuantía del 2015. 